Y. Yoshino

Nagoya Math. J.

Vol. 152 (1998), 39-56

\title{
TENSOR PRODUCTS OF MATRIX FACTORIZATIONS
}

\author{
YUJI YOSHINO
}

To the memory of Professor Hideyuki Matsumura

\begin{abstract}
Let $K$ be a field and let $f \in K\left[\left[x_{1}, x_{2}, \ldots, x_{r}\right]\right]$ and $g \in K\left[\left[y_{1}, y_{2}, \ldots\right.\right.$, $\left.\left.y_{s}\right]\right]$ be non-zero and non-invertible elements. If $X$ (resp. $Y$ ) is a matrix factorization of $f$ (resp. $g$ ), then we can construct the matrix factorization $X \widehat{\otimes} Y$ of $f+g$ over $K\left[\left[x_{1}, x_{2}, \ldots, x_{r}, y_{1}, y_{2}, \ldots, y_{s}\right]\right]$, which we call the tensor product of $X$ and $Y$.

After showing several general properties of tensor products, we will prove theorems which give bounds for the number of indecomposable components in the direct decomposition of $X \widehat{\otimes} Y$.
\end{abstract}

\section{$\S 0$. Introduction}

Let $K$ be a field and let $K[[x]]$ be the formal power series ring in the variables $x=x_{1}, x_{2}, \ldots, x_{r}$ and $K[[y]]$ the formal power series ring in $y=y_{1}, y_{2}, \ldots, y_{s}$. And let $f \in K[[x]]$ and $g \in K[[y]]$ be non-zero and noninvertible elements. We consider the problem how one can relate MCM modules (= maximal Cohen-Macaulay modules) over $R_{1}=K[[x]] /(f)$ and over $R_{2}=K[[y]] /(g)$ with MCM modules over $R=K[[x, y]] /(f+g)$. Actually if $R_{2}=K\left[\left[y_{1}\right]\right] /\left(y_{1}^{2}\right)$ or if $R_{2}=K\left[\left[y_{1}, y_{2}\right]\right] /\left(y_{1} y_{2}\right)$, then the problem was considered by Knörrer [K1], [K2], and a certain periodicity theorem for MCM modules is known in this course. (cf. [K2, Theorem 3.1].) On the other hand, Herzog and Popescu [HP] had considered the same problem for the particular case $R_{2}=K\left[\left[y_{1}\right]\right] /\left(y_{1}^{3}\right)$ and they call the problem "Thom-Sebastiani problem".

In this paper, we will present a method of tensor products of matrix factorizations to relate those objects. More precisely, if we have two MCM modules $M$ and $N$ respectively on the rings $R_{1}$ and $R_{2}$, we can construct a new MCM module $M \widehat{\otimes} N$ over $R$ by means of tensor product. In such a construction, it may happen that even if $M$ and $N$ are indecomposable, the tensor product $M \widehat{\otimes} N$ might be decomposable. Thus it will be a

Received December 25, 1996. 
major problem to find the condition for the indecomposability of the tensor modules, which is one of the main purposes of this paper.

In $\S 1$, we shall give a definition of the tensor product of matrix factorizations. Because every MCM module over a hypersurface ring is given by a matrix factorization, this formation will give the tensor product for MCM modules.

In $§ 2$, we give some functorial properties of tensor products, all of which are just machineries from the definition, however they will be useful for a practical computation.

In $\S 3$, we will find some of the criteria for the indecomposability of the tensor product. More precisely we will find some bounds for the numbers of indecomposable summands in the tensor product. See (3.3) and (3.4). Moreover, as one of the main theorems of this paper, we will show in (3.7) that if a matrix factorization $X$ of size one is not isomorphic to its first syzygy, then the tensor product by $X$ preserves the indecomposability.

In $\S 4$, an application of this theorem will be given, where we can claim that certain syzygy modules over a hypersurface ring are indecomposable.

\section{$\S 1$. Definition}

First we recall some of the basic facts about the relationship between MCM modules and matrix factorizations. Let $f \in K[[x]]=K\left[\left[x_{1}, x_{2}, \ldots, x_{r}\right]\right]$ be a non-zero and non-invertible element. A pair $(\phi, \psi)$ of $n \times n$-matrices over $K[[x]]$ is called a matrix factorization of $f$ of size $n$ if $\phi \psi=\psi \phi=f \cdot 1_{n}$.

If $(\phi, \psi)$ is a matrix factorization of $f$ of size $n$, then Eisenbud [E] shows that the module $M$ given by the exact sequence

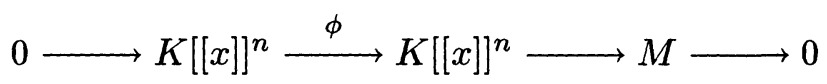

is always an MCM module over $K[[x]] /(f)$. Conversely every MCM module over $K[[x]] /(f)$ is given in this way. More precisely, there is a stable equivalence of the category of MCM modules with the category of matrix factorizations. (See $[\mathrm{E}]$ or $[\mathrm{Y} 1,(7.4)]$.)

We denote the category of matrix factorizations of $f$ by $M F_{K[[x]]}(f)$ which is defined as follows: The objects of $M F_{K[[x]]}(f)$ are the matrix factorizations of $f$, and the morphisms from $\left(\phi_{1}, \psi_{1}\right)$ to $\left(\phi_{2}, \psi_{2}\right)$ are the 
pairs of matrices $(\alpha, \beta)$ which make the following diagram commutative:

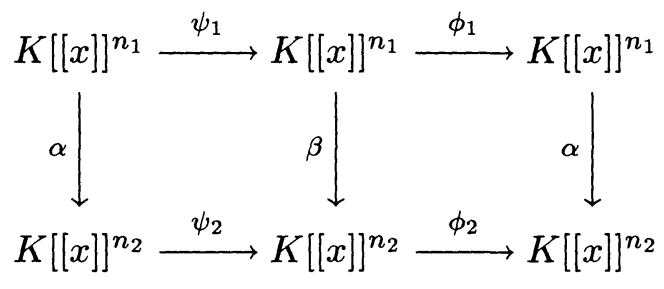

We quote the following fact from Solberg [S, Prop. 1.3].

LEMMA 1.1. The category $M F_{K[[x]]}(f)$ is equivalent to the category of $M C M$ modules over a certain (noncommutative) order on $K[[x]]$. In particular, $M F_{K[[x]]}(f)$ is an additive category with splitting idempotent property. Therefore the Krull-Schmidt theorem holds in $M F_{K[[x]]}(f)$.

Since, in this paper, we only consider MCM modules over hypersurface rings, we mainly concentrate on the matrix factorizations.

Now to define the tensor products of matrix factorizations, as in the introduction let $f \in K[[x]]=K\left[\left[x_{1}, x_{2}, \ldots, x_{r}\right]\right]$ and $g \in K[[y]]=K\left[\left[y_{1}, y_{2}, \ldots\right.\right.$, $\left.y_{s}\right]$ be two formal power series that are non-zero and non-invertible.

In the following $X=(\phi, \psi)$ (resp. $\left.X^{\prime}=\left(\phi^{\prime}, \psi^{\prime}\right)\right)$ is always an object in $M F_{K[[x]]}(f)\left(\right.$ resp. $\left.M F_{K[[y]]}(g)\right)$ of size $n$ (resp. $m$ ).

Definition 1.2. Since $\phi$ and $\psi$ (resp. $\phi^{\prime}$ and $\psi^{\prime}$ ) are matrices over $K[[x]]$ (resp. $K[[y]]$ ), they can be considered as matrices over $S:=K[[x, y]]$ of size $n$ (resp. $m$ ). Then the tensor product $X \widehat{\otimes} X^{\prime}$ is given by

$$
\left(\left(\begin{array}{cc}
\phi \otimes 1_{m} & 1_{n} \otimes \phi^{\prime} \\
-1_{n} \otimes \psi^{\prime} & \psi \otimes 1_{m}
\end{array}\right),\left(\begin{array}{cc}
\psi \otimes 1_{m} & -1_{n} \otimes \phi^{\prime} \\
1_{n} \otimes \psi^{\prime} & \phi \otimes 1_{m}
\end{array}\right)\right)
$$

where each component is an endomorphism on $S^{n} \otimes_{S} S^{m}$. It is easy to verify that $X \widehat{\otimes} X^{\prime}$ is in fact an object of $M F_{S}(f+g)$ of size $2 n m$.

For a morphism $\xi=(\alpha, \beta): X_{1}=\left(\phi_{1}, \psi_{1}\right) \rightarrow X_{2}=\left(\phi_{2}, \psi_{2}\right)$ in $M F_{K[[x]]}(f)$, we also define $\xi \widehat{\otimes} X^{\prime}$ by

$$
\left(\left(\begin{array}{cc}
\alpha \otimes 1_{m} & 0 \\
0 & \beta \otimes 1_{m}
\end{array}\right),\left(\begin{array}{cc}
\beta \otimes 1_{m} & 0 \\
0 & \alpha \otimes 1_{m}
\end{array}\right)\right)
$$

Actually $\xi \widehat{\otimes} X^{\prime}$ is a morphism $X_{1} \widehat{\otimes} X^{\prime} \rightarrow X_{2} \widehat{\otimes} X^{\prime}$ in $M F_{S}(f+g)$. 
Similarly, if $\xi^{\prime}=\left(\alpha^{\prime}, \beta^{\prime}\right): X_{1}^{\prime}=\left(\phi_{1}^{\prime}, \psi_{1}^{\prime}\right) \rightarrow X_{2}^{\prime}=\left(\phi_{2}^{\prime}, \psi_{2}^{\prime}\right)$ is a morphism in $M F_{K[[y]]}(g)$, then $X \widehat{\otimes} \xi^{\prime}$ is defined to be

$$
\left(\left(\begin{array}{cc}
1_{n} \otimes \alpha^{\prime} & 0 \\
0 & 1_{n} \otimes \beta^{\prime}
\end{array}\right)\left(\begin{array}{cc}
1_{n} \otimes \alpha^{\prime} & 0 \\
0 & 1_{n} \otimes \beta^{\prime}
\end{array}\right)\right) .
$$

Thus for fixed $X$ and $X^{\prime}$, the tensor product $X \widehat{\otimes}\left(\right.$ ) (resp. ( ) $\widehat{\otimes} X^{\prime}$ ) defines the functor $M F_{K[[y]]}(g) \rightarrow M F_{S}(f+g)\left(\operatorname{resp.} M F_{K[[x]]}(f) \rightarrow M F_{S}(f+g)\right)$.

Remark 1.3. (i) If $g\left(y_{1}\right)=y_{1}^{2}$ and $X^{\prime}=\left(y_{1}, y_{1}\right)$ or if $g(y)=y_{1} y_{2}$ and $X^{\prime}=\left(y_{1}, y_{2}\right)$, then the tensor product ()$\widehat{\otimes} X^{\prime}$ coincides with the functor defined by Knörrer [K2, $\S 2$ and $\S 3]$.

(ii) If both $f$ and $g$ are quadratic forms, then the above formation of tensor products just corresponds to the tensor products of Clifford modules. See $[\mathrm{BEH}]$ or [Y1, Chap. 14].

EXAMPLE 1.4. Suppose we have three matrix factorizations in one variable: $X=\left(x, x^{2}\right) \in M F_{K[[x]]}\left(x^{3}\right), X^{\prime}=\left(y^{2}, y^{3}\right) \in M F_{K[[y]]}\left(y^{5}\right)$ and $X^{\prime \prime}=\left(z^{3}, z^{4}\right) \in M F_{K[[z]]}\left(z^{7}\right)$. Setting $Y=X \widehat{\otimes} X^{\prime}$, we have

$$
Y=\left(\left(\begin{array}{cc}
x & y^{2} \\
-y^{3} & x^{2}
\end{array}\right),\left(\begin{array}{cc}
x^{2} & -y^{2} \\
y^{3} & x
\end{array}\right)\right)
$$

Therefore we get a matrix factorization $Y \widehat{\otimes} X^{\prime \prime} \in M F_{K[[x, y, z]]}\left(x^{3}+y^{5}+z^{7}\right)$ described as follows:

$$
Y \widehat{\otimes} X^{\prime \prime}=\left(\left(\begin{array}{cccc}
x & y^{2} & z^{3} & 0 \\
-y^{3} & x^{2} & 0 & z^{3} \\
-z^{4} & 0 & x^{2} & -y^{2} \\
0 & -z^{4} & y^{3} & x
\end{array}\right),\left(\begin{array}{cccc}
x^{2} & -y^{2} & -z^{3} & 0 \\
y^{3} & x & 0 & -z^{3} \\
z^{4} & 0 & x & y^{2} \\
0 & z^{4} & -y^{3} & x^{2}
\end{array}\right)\right)
$$

We shall show in (3.7) that $Y \widehat{\otimes} X^{\prime \prime}$ is indecomposable.

\section{$\S 2$. Functorial properties}

We show in this section the basic properties of tensor products. As in $\S 1, X=(\phi, \psi) \in M F_{K[[x]]}(f), X^{\prime}=\left(\phi^{\prime}, \psi^{\prime}\right) \in M F_{K[[y]]}(g)$ always denote the matrix factorizations of size $n$ and $m$ respectively and set $S=K[[x, y]]$.

LEMMA 2.1. (Commutativity) There is a natural isomorphism $X \widehat{\otimes}$ $X^{\prime} \cong X^{\prime} \widehat{\otimes} X$ in $M F_{S}(f+g)$. 
Proof. This is obvious from the following commutative diagram:

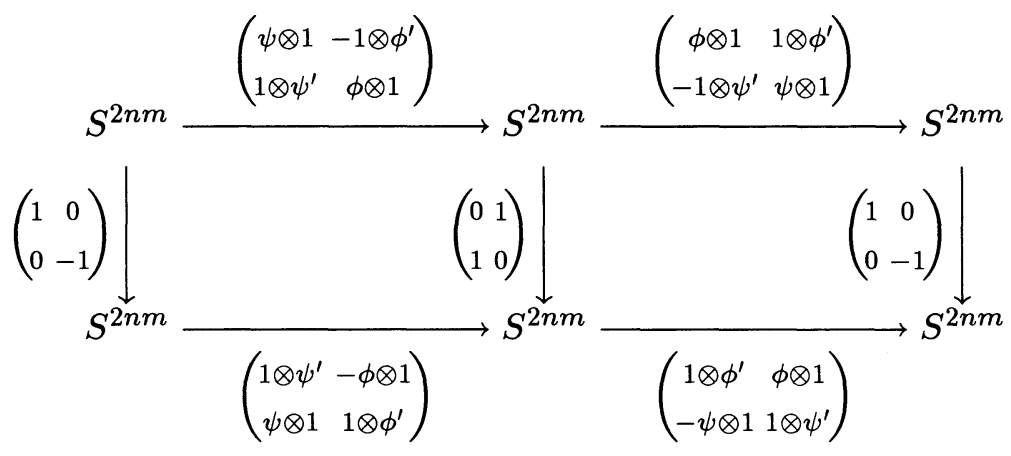

By virtue of this lemma, if we prove some claim for the variable $X$, the same will be valid for the variable $X^{\prime}$. (E.g. Lemmas (2.2), (2.3), (2.8), (2.11) and (2.12) below.)

LEMMA 2.2. (Additivity) There is a natural isomorphism $\left(X_{1} \oplus X_{2}\right) \widehat{\otimes}$ $X^{\prime} \cong\left(X_{1} \widehat{\otimes} X^{\prime}\right) \oplus\left(X_{2} \widehat{\otimes} X^{\prime}\right)$.

Proof. Let $X_{1}=\left(\phi_{1}, \psi_{1}\right)$ and $X_{2}=\left(\phi_{2}, \psi_{2}\right)$ be of size $n_{1}$ and $n_{2}$ respectively. Then we have

$$
\begin{aligned}
& \left(X_{1} \oplus X_{2}\right) \widehat{\otimes} X^{\prime} \\
& =\left(\left(\begin{array}{cccc}
\phi_{1} \otimes 1 & & 1 \otimes \phi^{\prime} & \\
& \phi_{2} \otimes 1 & & 1 \otimes \phi^{\prime} \\
-1 \otimes \psi^{\prime} & & \psi_{1} \otimes 1 & \\
& -1 \otimes \psi^{\prime} & & \psi_{2} \otimes 1
\end{array}\right),\left(\begin{array}{cccc}
\psi_{1} \otimes 1 & & -1 \otimes \phi^{\prime} & \\
& \psi_{2} \otimes 1 & & -1 \otimes \phi^{\prime} \\
1 \otimes \psi^{\prime} & & \phi_{1} \otimes 1 & \\
& 1 \otimes \psi^{\prime} & & \phi_{2} \otimes
\end{array}\right)\right)
\end{aligned}
$$

and

$$
\begin{aligned}
& \left(X_{1} \widehat{\otimes} X^{\prime}\right) \oplus\left(X_{2} \widehat{\otimes} X^{\prime}\right) \\
& =\left(\left(\begin{array}{cccc}
\phi_{1} \otimes 1 & 1 \otimes \phi^{\prime} & & \\
-1 \otimes \psi^{\prime} & \psi_{1} \otimes 1 & & \\
& & \phi_{2} \otimes 1 & 1 \otimes \phi^{\prime} \\
& & -1 \otimes \psi^{\prime} & \psi_{2} \otimes 1
\end{array}\right),\left(\begin{array}{cccc}
\psi_{1} \otimes 1 & -1 \otimes \phi^{\prime} & & \\
1 \otimes \psi^{\prime} & \phi_{1} \otimes 1 & & \\
& & \psi_{2} \otimes 1 & -1 \otimes \phi^{\prime} \\
& & 1 \otimes \psi^{\prime} & \phi_{2} \otimes 1
\end{array}\right)\right) .
\end{aligned}
$$

From these equalities the lemma follows easily.

A matrix factorization $X \in M F_{K[[x]]}(f)$ is called trivial if $X$ is a direct sum of copies of the matrix factorizations $(f, 1)$ and $(1, f)$. 
LEMMA 2.3. (Preserving triviality) If $X$ is a trivial matrix factorization in $M F_{K[[x]]}(f)$, then $X \widehat{\otimes} X^{\prime}$ is also a trivial matrix factorization in $M F_{S}(f+g)$.

Proof. If $X=(1, f)$, then we see that

$$
\begin{aligned}
X \widehat{\otimes} X^{\prime} & =\left(\left(\begin{array}{cc}
1 \otimes 1_{m} & 1 \otimes \phi^{\prime} \\
-1 \otimes \psi^{\prime} & f \otimes 1_{m}
\end{array}\right),\left(\begin{array}{cc}
f \otimes 1_{m} & -1 \otimes \phi^{\prime} \\
1 \otimes \psi^{\prime} & 1 \otimes 1_{m}
\end{array}\right)\right) \\
& \left.\cong\left(\begin{array}{cc}
1 \otimes 1_{m} & 1 \otimes \phi^{\prime} \\
0 & (f+g)\left(1 \otimes 1_{m}\right)
\end{array}\right),\left(\begin{array}{cc}
(f+g)\left(1 \otimes 1_{m}\right) & -1 \otimes \phi^{\prime} \\
0 & 1 \otimes 1_{m}
\end{array}\right)\right) \\
& \left.\cong\left(\begin{array}{cc}
1 \otimes 1_{m} & \\
& (f+g)\left(1 \otimes 1_{m}\right)
\end{array}\right),\left(\begin{array}{cc}
(f+g)\left(1 \otimes 1_{m}\right) & \\
& 1 \otimes 1_{m}
\end{array}\right)\right),
\end{aligned}
$$

hence $X \widehat{\otimes} X^{\prime} \cong(f+g, 1)^{m} \oplus(1, f+g)^{m}$ is trivial. The same is true for $X=(f, 1)$ and the lemma follows from (2.2).

From (2.2) and (2.3) we have the additive functor between stable categories

$$
\left(\text { ) } \widehat{\otimes} X^{\prime}: \underline{M F}_{K[[x]]}(f) \rightarrow \underline{M F}_{S}(f+g) .\right.
$$

See [Y1, Chap. 7] for the definition of the stable categories.

For a matrix factorization $(\phi, \psi)$, the syzygy $\Omega(\phi, \psi)$ is defined to be the matrix factorization $(\psi, \phi)$. Notice that $\Omega$ defines the endo-functor on the category of matrix factorizations and satisfies $\Omega^{2}=1$.

LEMma 2.5. (Syzygy property) There are isomorphisms in $M F_{S}(f+$ $g)$ :

$$
\Omega X \widehat{\otimes} \Omega X^{\prime} \cong X \widehat{\otimes} X^{\prime}, \quad X \widehat{\otimes} \Omega X^{\prime} \cong \Omega\left(X \widehat{\otimes} X^{\prime}\right) \cong \Omega X \widehat{\otimes} X^{\prime} .
$$

Proof. The first isomorphism follows from the following commutative diagram:

$$
\begin{aligned}
& S^{2 n m} \stackrel{\left(\begin{array}{cc}
\phi \otimes 1 & -1 \otimes \psi^{\prime} \\
1 \otimes \phi^{\prime} & \psi \otimes 1
\end{array}\right)}{\longrightarrow} S^{2 n m} \stackrel{\left(\begin{array}{cc}
\psi \otimes 1 & 1 \otimes \psi^{\prime} \\
-1 \otimes \phi^{\prime} & \phi \otimes 1
\end{array}\right)}{\longrightarrow} S^{2 n m} \\
& \left(\begin{array}{cc}
0 & 1 \\
-1 & 0
\end{array}\right) S_{S^{2 n m}}^{\underset{\left(\begin{array}{cc}
\psi \otimes 1 & -1 \otimes \phi^{\prime} \\
1 \otimes \psi^{\prime} & \phi \otimes 1
\end{array}\right)}{\longrightarrow}} S^{2 n m} \underset{\left(\begin{array}{cc}
0 & 1 \\
-1 & 0
\end{array}\right) \mid}{\left(\begin{array}{cc}
\phi \otimes 1 & 1 \otimes \phi^{\prime} \\
-1 \otimes \psi^{\prime} & \psi \otimes 1
\end{array}\right)} S^{2 n m}
\end{aligned}
$$


We omit the proof of the rest because they are just the consequences of direct computations similar to this.

Note in general $\Omega\left(X \widehat{\otimes} X^{\prime}\right) \not X \widehat{\otimes} X^{\prime}$.

For a matrix factorization $X=(\phi, \psi)$ we define the dual $X^{*}$ of $X$ by the transposes of matrices; $X^{*}=\left(\phi^{t}, \psi^{t}\right)$. Note that this operation corresponds to the canonical dual in the category of MCM modules.

By a similar computation as in the previous lemma, we can show the following

LEMmA 2.6. (Dual) There is an isomorphism $\left(X \widehat{\otimes} X^{\prime}\right)^{*} \cong X^{*} \widehat{\otimes} X^{\prime *}$.

LEMMA 2.7. (Associativity) As before let $X=(\phi, \psi) \in M F_{K[[x]]}(f)$ and $X^{\prime}=\left(\phi^{\prime}, \psi^{\prime}\right) \in M F_{K[[y]]}(g)$. Further suppose $X^{\prime \prime}=\left(\phi^{\prime \prime}, \psi^{\prime \prime}\right)$ is in $M F_{K[[z]]}(h)$. Then we have an isomorphism in $M F(f+g+h)$ :

$$
\left(X \widehat{\otimes} X^{\prime}\right) \widehat{\otimes} X^{\prime \prime} \cong X \widehat{\otimes}\left(X^{\prime} \widehat{\otimes} X^{\prime \prime}\right)
$$

Proof. For simplicity we denote $\phi^{(1)}=\phi \otimes 1 \otimes 1, \phi^{(2)}=1 \otimes \phi^{\prime} \otimes 1$, $\phi^{(3)}=1 \otimes 1 \otimes \phi^{\prime \prime}$, and similarly $\psi^{(1)}=\psi \otimes 1 \otimes 1, \psi^{(2)}=1 \otimes \psi^{\prime} \otimes 1$, $\psi^{(3)}=1 \otimes 1 \otimes \psi^{\prime \prime}$. Then an easy computation shows that

$\left(X \widehat{\otimes} X^{\prime}\right) \widehat{\otimes} X^{\prime \prime}$

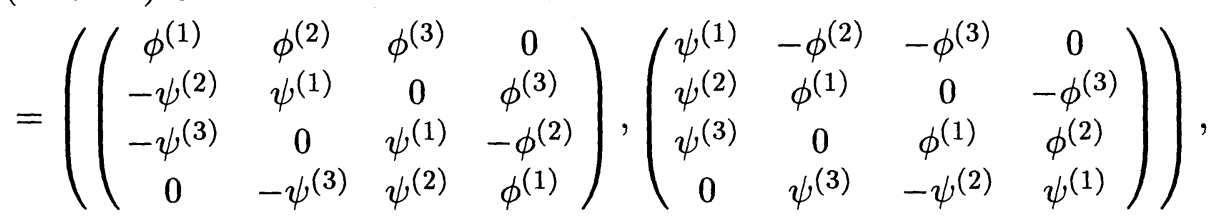

and

$$
\begin{aligned}
& X \widehat{\otimes}\left(X^{\prime} \widehat{\otimes} X^{\prime \prime}\right) \\
& \left.=\left(\begin{array}{cccc}
\phi^{(1)} & 0 & \phi^{(2)} & \phi^{(3)} \\
0 & \phi^{(1)} & -\psi^{(3)} & \psi^{(2)} \\
-\psi^{(2)} & \phi^{(3)} & \psi^{(1)} & 0 \\
-\psi^{(3)} & -\phi^{(2)} & 0 & \psi^{(1)}
\end{array}\right),\left(\begin{array}{cccc}
\psi^{(1)} & 0 & -\phi^{(2)} & -\phi^{(3)} \\
0 & \psi^{(1)} & \psi^{(3)} & -\psi^{(2)} \\
\psi^{(2)} & -\phi^{(3)} & \phi^{(1)} & 0 \\
\psi^{(3)} & \phi^{(2)} & 0 & \phi^{(1)}
\end{array}\right)\right) .
\end{aligned}
$$

Then changing rows and columns results the isomorphism in the lemma.

We say that the matrix factorization is reduced if no entries of the matrices are units. 
Lemma 2.8. (Exactness) If $(i) 0 \rightarrow X_{1} \rightarrow X_{2} \rightarrow X_{3} \rightarrow 0$ is an exact sequence in $M F_{K[[x]]}(f)$, then it induces the following exact sequence in $M F_{S}(f+g)$ :

$$
0 \longrightarrow X_{1} \widehat{\otimes} X^{\prime} \longrightarrow X_{2} \widehat{\otimes} X^{\prime} \longrightarrow X_{3} \widehat{\otimes} X^{\prime} \longrightarrow 0
$$

Furthermore if the sequence (ii) splits and if $X^{\prime}$ is a reduced matrix factorization, then the first sequence $(i)$ also splits.

Proof. Let $\xi=(\alpha, \beta) \in \operatorname{Ext}^{1}\left(X_{3}, X_{1}\right) \cong \underline{\operatorname{Hom}}\left(X_{3}, \Omega X_{1}\right)$ be an element corresponding to the extension $(i)$. Then the matrix factorization of $f$ given by $E=\left(\left(\begin{array}{cc}\phi_{1} & \beta \\ & -\phi_{3}\end{array}\right),\left(\begin{array}{cc}\psi_{1} & \alpha \\ & -\psi_{3}\end{array}\right)\right)$ represents $X_{2}$ in $(i)$. Thus $X_{2} \widehat{\otimes} X^{\prime}$ is represented by

$$
\begin{aligned}
& E \widehat{\otimes} X^{\prime} \\
& \begin{array}{l}
=\left(\left(\begin{array}{cccc}
\phi_{1} \otimes 1 & \beta \otimes 1 & 1 \otimes \phi^{\prime} & \\
& -\phi_{3} \otimes 1 & & 1 \otimes \phi^{\prime} \\
-1 \otimes \psi^{\prime} & & \psi_{1} \otimes 1 & \alpha \otimes 1 \\
& -1 \otimes \psi^{\prime} & & -\psi_{3} \otimes 1
\end{array}\right),\left(\begin{array}{cccc}
\psi_{1} \otimes 1 & \alpha \otimes 1 & -1 \otimes \phi^{\prime} & \\
& -\psi_{3} \otimes 1 & & -1 \otimes \phi^{\prime} \\
1 \otimes \psi^{\prime} & & \phi_{1} \otimes 1 & \beta \otimes 1 \\
& 1 \otimes \psi^{\prime} & & -\phi_{3} \otimes 1
\end{array}\right)\right) \\
\left.\cong\left(\begin{array}{cccc}
\phi_{1} \otimes 1 & 1 \otimes \phi^{\prime} & \beta \otimes 1 & \\
-1 \otimes \psi^{\prime} & \psi_{1} \otimes 1 & & \alpha \otimes 1 \\
& & -\phi_{3} \otimes 1 & 1 \otimes \phi^{\prime} \\
& & -1 \otimes \psi^{\prime} & -\psi_{3} \otimes 1
\end{array}\right),\left(\begin{array}{cccc}
\psi_{1} \otimes 1 & -1 \otimes \phi^{\prime} & \alpha \otimes 1 & \\
1 \otimes \psi^{\prime} & \phi_{3} \otimes 1 & & \beta \otimes 1 \\
& & -\psi_{3} \otimes 1 & -1 \otimes \phi^{\prime} \\
& & -1 \otimes \psi^{\prime} & -\phi_{3} \otimes 1
\end{array}\right)\right)
\end{array}
\end{aligned}
$$

This shows that the sequence $(i i)$ is an extension represented by $\xi \widehat{\otimes} X^{\prime} \in$ $\underline{\operatorname{Hom}}\left(X_{3} \widehat{\otimes} X^{\prime}, \Omega X_{1} \widehat{\otimes} X^{\prime}\right)$, in particular it is exact.

If $(i i)$ splits, then $(i i) \otimes_{S} S /(y)$ also splits, and we can use the next lemma 2.9 to get the following sequence splits in $M F_{K[[x]]}(f)$ :

$$
0 \longrightarrow\left(X_{1} \oplus \Omega X_{1}\right)^{m} \longrightarrow\left(X_{2} \oplus \Omega X_{2}\right)^{m} \longrightarrow\left(X_{3} \oplus \Omega X_{3}\right)^{m} \longrightarrow 0
$$

Therefore $(i)$ also splits.

DeFinition AND LEMMA 2.9. (Reduction) If $Y=(\Phi, \Psi) \in M F_{S}(f+$ $g$ ), then the pair of matrices $\left(\Phi \otimes_{S} S /(y), \Psi \otimes_{S} S /(y)\right)$ gives a matrix factorization of $f$ in $M F_{K[[x]]}(f)$. Thus ()$\otimes_{S} S /(y)$ defines a functor $M F_{S}(f+g) \rightarrow M F_{K[[x]]}(f)$, which we call the reduction to $S /(y)$ and denote by ()$_{y}$. 
Under this notation, if $X^{\prime}$ is a reduced matrix factorization of size $m$, then we have a functorial isomorphism $\left(X \widehat{\otimes} X^{\prime}\right)_{y} \cong(X \oplus \Omega X)^{m}$ in $M F_{K[[x]]}(f)$.

Similarly if $X$ is reduced of size $n$, then the reduction to $S /(x)$ gives the isomorphism $\left(X \widehat{\otimes} X^{\prime}\right)_{x} \cong\left(X^{\prime} \oplus \Omega X^{\prime}\right)^{n}$

Proof. The first part of the lemma is obvious. For the second, notice that $\phi^{\prime} \otimes S /(y)=\psi^{\prime} \otimes S /(y)=0$, therefore we have the following equalities for an object $X=(\phi, \psi)$ in $M F_{K[[x]]}(f)$ :

$$
\begin{aligned}
\left(X \widehat{\otimes} X^{\prime}\right)_{y} & =\left(\left(\begin{array}{ll}
\phi \otimes 1 & \\
& \psi \otimes 1
\end{array}\right),\left(\begin{array}{ll}
\psi \otimes 1 & \\
& \phi \otimes 1
\end{array}\right)\right) \\
& =(\phi, \psi)^{m} \oplus(\psi, \phi)^{m} .
\end{aligned}
$$

The lemma follows from this equality.

LEMMA 2.10. Let $X^{\prime}$ be a reduced matrix factorization in $M F(g)$ as above and let $\xi$ be a morphism $X_{1} \widehat{\otimes} X^{\prime} \rightarrow X_{2} \widehat{\otimes} X^{\prime}$ in $M F(f+g)$. Then we can describe $\xi$ as follows:

$$
\xi=\left(\left(\begin{array}{ll}
\alpha & \beta \\
\gamma & \delta
\end{array}\right),\left(\begin{array}{ll}
\alpha^{\prime} & \beta^{\prime} \\
\gamma^{\prime} & \delta^{\prime}
\end{array}\right)\right),
$$

where $\alpha, \alpha^{\prime}, \beta$ etc. are $n m \times n m$ matrices over $S$. In this case if we take the reduction to $S /(y)$, we have the morphism $\xi_{y}: X_{1}^{m} \oplus \Omega X_{1}^{m} \rightarrow X_{2}^{m} \oplus \Omega X_{2}^{m}$ described as

$$
\left(\begin{array}{cc}
\left(\alpha, \alpha^{\prime}\right)_{y} & \left(\beta, \beta^{\prime}\right)_{y} \\
\left(\gamma, \gamma^{\prime}\right)_{y} & \left(\delta, \delta^{\prime}\right)_{y}
\end{array}\right) .
$$

Similarly, let $X \in M F(f)$ be reduced and let $\eta$ be a morphism $X \widehat{\otimes} X_{1}^{\prime} \rightarrow$ $X \widehat{\otimes} X_{2}^{\prime}$ in $M F(f+g)$. As above, if we write $\eta$ as

$$
\eta=\left(\left(\begin{array}{ll}
\alpha & \beta \\
\gamma & \delta
\end{array}\right),\left(\begin{array}{ll}
\alpha^{\prime} & \beta^{\prime} \\
\gamma^{\prime} & \delta^{\prime}
\end{array}\right)\right)
$$

then taking the reduction to $S /(x)$ we have a morphism $\eta_{x}: X_{1}^{\prime n} \oplus \Omega X_{1}^{\prime n} \rightarrow$ $X_{2}^{\prime n} \oplus \Omega X_{2}^{\prime n}$ described as

$$
\left(\begin{array}{cc}
\left(\alpha, \delta^{\prime}\right)_{x} & \left(\beta,-\gamma^{\prime}\right)_{x} \\
\left(\gamma,-\beta^{\prime}\right)_{x} & \left(\delta, \alpha^{\prime}\right)_{x}
\end{array}\right)
$$


Proof. We prove the lemma only for $\eta$. From the definition we know the equality

$$
\left(\begin{array}{cc}
\alpha & \beta \\
\gamma & \delta
\end{array}\right)\left(\begin{array}{cc}
\phi \otimes 1 & 1_{n} \otimes \phi_{1}^{\prime} \\
-1_{n} \otimes \psi_{1}^{\prime} & \psi \otimes 1
\end{array}\right)=\left(\begin{array}{cc}
\phi \otimes 1 & 1_{n} \otimes \phi_{2}^{\prime} \\
-1_{n} \otimes \psi_{2}^{\prime} & \psi \otimes 1
\end{array}\right)\left(\begin{array}{cc}
\alpha^{\prime} & \beta^{\prime} \\
\gamma^{\prime} & \delta^{\prime}
\end{array}\right),
$$

as matrices over $S$. Note that $(\phi \otimes 1)_{x}=(\psi \otimes 1)_{x}=0,\left(1_{n} \otimes \zeta^{\prime}\right)_{x}=\zeta^{\prime n}$ for any matrix $\zeta^{\prime}$ over $K[[y]]$, where $\zeta^{\prime n}$ denotes the direct sum of $n$ copies of $\zeta^{\prime}$. It hence follows that $\alpha_{x}\left(\phi_{1}^{\prime n}\right)=\left(\phi_{2}^{\prime n}\right) \delta_{x}^{\prime},-\beta_{x}\left(\psi_{1}^{\prime n}\right)=\left(\phi_{2}^{\prime n}\right) \gamma_{x}^{\prime}, \delta_{x}\left(\psi_{1}^{\prime n}\right)=$ $\left(\psi_{2}^{\prime n}\right) \alpha_{x}^{\prime}$ and $\gamma_{x}\left(\phi_{1}^{\prime n}\right)=-\left(\psi_{2}^{\prime n}\right) \beta_{x}^{\prime}$. This shows that we have the morphisms $\left(\alpha, \delta^{\prime}\right)_{x}: X_{1}^{\prime n} \rightarrow X_{2}^{\prime n},\left(\beta,-\gamma^{\prime}\right)_{x}: \Omega X_{1}^{\prime n} \rightarrow X_{2}^{\prime n},\left(\delta, \alpha^{\prime}\right)_{x}: \Omega X_{1}^{\prime n} \rightarrow \Omega X_{2}^{\prime n}$ and $\left(\gamma,-\beta^{\prime}\right)_{x}: X_{1}^{\prime n} \rightarrow \Omega X_{2}^{\prime n}$.

As an application of Lemma (2.9) we see the following

LEMMA 2.11. (Faithfulness) If $X^{\prime}$ is a reduced matrix factorization, then the functor $(\quad) \widehat{\otimes} X^{\prime}$ in (2.4) is faithful, that is, the induced map $\underline{\operatorname{Hom}}\left(X_{1}, X_{2}\right) \longrightarrow \underline{\operatorname{Hom}}\left(X_{1} \widehat{\otimes} X^{\prime}, X_{2} \widehat{\otimes} X^{\prime}\right)$ is injective.

One has to notice that the functor is far from "full."

Proof. Let $\xi: X_{1} \rightarrow X_{2}$ be a morphism in $M F_{K[[x]]}(f)$ and suppose that $\xi \widehat{\otimes} X^{\prime}$ factors through a trivial matrix factorization $Y \in M F_{S}(f+g)$. Note that $Y_{y}$ is also a trivial matrix factorization in $M F_{K[[x]]}(f)$, and we see from $(2.9)$ that $\left(\xi \widehat{\otimes} X^{\prime}\right)_{y}=(\xi \oplus \Omega \xi)^{m}$ also factors through a trivial matrix factorization. Thus $(\xi \oplus \Omega \xi)^{m}=0$ in $\underline{M F}_{K[[x]]}(f)$, and $\xi=0$ in $\underline{M F}_{K[[x]]}(f)$.

LEMmA 2.12. Suppose that $X^{\prime} \in M F_{K[[y]]}(g)$ is reduced.

(i) For a morphism $\xi$ in $M F_{K[[x]]}(f)$, if $\xi \widehat{\otimes} X^{\prime}$ is an isomorphism in $M F_{S}(f+g)$, then $\xi$ is also an isomorphism.

(ii) For indecomposable objects $X_{1}$ and $X_{2}$ in $M F_{K[[x]]}(f)$, if $X_{1} \widehat{\otimes} X^{\prime} \cong$ $X_{2} \widehat{\otimes} X^{\prime}$ in $M F_{S}(f+g)$, then $X_{1} \cong X_{2}$ or $X_{1} \cong \Omega X_{2}$ in $M F_{K[[x]]}(f)$.

Proof. (i) If $\xi \widehat{\otimes} X^{\prime}$ is an isomorphism, then $\left(\xi \widehat{\otimes} X^{\prime}\right)_{y}=(\xi \oplus \Omega \xi)^{m}$ is also an isomorphism, hence is $\xi$.

(ii) It follows from the assumption and from (2.9) that $\left(X_{1} \oplus \Omega X_{1}\right)^{m} \cong$ $\left(X_{2} \oplus \Omega X_{2}\right)^{m}$. Since $X_{1}$ and $X_{2}$ are indecomposable, from (1.1) we have either $X_{1} \cong X_{2}$ or $X_{1} \cong \Omega X_{2}$.

Remark 2.13. There is an example satisfying $X \not \Omega X$ and $X \widehat{\otimes} X^{\prime} \cong$ $\Omega X \widehat{\otimes} X^{\prime}$. For example, $X=\left(x_{1}, x_{2}\right) \in M F\left(x_{1} x_{2}\right)$ and $X^{\prime}=(y, y) \in$ $M F\left(y^{2}\right)$. 


\section{$\S 3$. Decomposition of Tensor Products}

In the rest of the paper we use the following

Notation 3.1. Let $X=(\phi, \psi) \in M F_{K[[x]]}(f)$ and $X^{\prime}=\left(\phi^{\prime}, \psi^{\prime}\right) \in$ $M F_{K[[y]]}(g)$ be indecomposable reduced matrix factorizations of size $n, m$ respectively. We denote the number of indecomposable summands in the direct decomposition of $X \widehat{\otimes} X^{\prime}$ by $\#\left(X \widehat{\otimes} X^{\prime}\right)$. And let $r=(n, m)$ be the greatest common divisor of $n$ and $m$.

Under these notation, we are interested in how the tensor product $X \widehat{\otimes}$ $X^{\prime}$ decomposes or when it is indecomposable. We begin with the following fact.

LEMMA 3.2. Suppose the characteristic of the field $K$ is unequal to 2 and that $K$ contains the square root $i$ of -1 . And assume that $X=$ $(\phi, \phi)$ and $X^{\prime}=\left(\phi^{\prime}, \phi^{\prime}\right)$ (so that $\psi=\phi, \psi^{\prime}=\phi^{\prime}$ or equivalently $\Omega X=X$, $\Omega X^{\prime}=X^{\prime}$.) Then $X \widehat{\otimes} X^{\prime}$ is decomposable as $X \widehat{\otimes} X^{\prime} \cong Y \oplus \Omega Y$ where $Y=\left(\phi \otimes 1_{m}-i\left(1_{n} \otimes \phi^{\prime}\right), \phi \otimes 1_{m}+i\left(1_{n} \otimes \phi^{\prime}\right)\right)$.

Proof. This is clear from the following equality:

$$
\begin{aligned}
\left(\begin{array}{cc}
1 & i \\
\frac{1}{2} i & \frac{1}{2}
\end{array}\right) & \left(\begin{array}{cc}
\phi \otimes 1 & 1 \otimes \phi^{\prime} \\
-1 \otimes \phi^{\prime} & \phi \otimes 1
\end{array}\right)\left(\begin{array}{cc}
\frac{1}{2} & -i \\
-\frac{1}{2} i & 1
\end{array}\right) \\
= & \left(\begin{array}{cc}
\phi \otimes 1-i\left(1 \otimes \phi^{\prime}\right) & 0 \\
0 & \phi \otimes 1+i\left(1 \otimes \phi^{\prime}\right)
\end{array}\right)
\end{aligned}
$$

For the number of indecomposable factors we have the following rather rough estimation.

THEOREM 3.3. There is an inequality $\#\left(X \widehat{\otimes} X^{\prime}\right) \leqq 2 r$.

Proof. We claim that any summand $Y$ of $X \widehat{\otimes} X^{\prime}$ has size at least $n m / r$. If we have shown this, then since $X \widehat{\otimes} X^{\prime}$ has size $2 n m$, it has at most $2 r$ summands.

To prove the above, notice from (2.9) that $Y_{y}$ is a direct summand of $(X \oplus \Omega X)^{m}$. Since both of $X$ and $\Omega X$ are indecomposable, it follows from (1.1) that $Y_{y} \cong X^{k} \oplus \Omega X^{\ell}$ for some $0 \leqq k, \ell \leqq m$. Since the reduction does not change the size of a matrix factorization, we can write the size of $Y$ as $(k+\ell) n$. Similarly, considering the reduction to $S /(x)$, we have that the size of $Y$ equals to $(p+q) m$ for some $0 \leqq p, q \leqq n$. Hence $(k+\ell) m=(p+q) n$ which must be $\geqq \operatorname{lcm}(n, m)=m n / r$. 


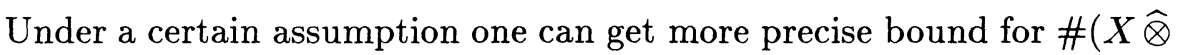
$\left.X^{\prime}\right)$ as follows.

THEOREM 3.4. If $\Omega X \not X$ and if $\Omega X^{\prime} \not X^{\prime}$, then $\#\left(X \widehat{\otimes} X^{\prime}\right) \leqq r$.

To prove this we need a lemma.

Lemma 3.5. Let $\mathcal{C}$ be a Krull-Schmidt category with splitting idempotent property. Furthermore let $A$ and $B$ be indecomposable objects in $\mathcal{C}$ and $X=A^{n} \oplus B^{m}$. Suppose $e=\left(\begin{array}{ll}a & b \\ c & d\end{array}\right) \in \operatorname{End}(X)=\operatorname{End}\left(A^{n} \oplus B^{m}\right)$ is an idempotent.

(i) If $A$ is not isomorphic to $B$, then $b c \in \operatorname{rad} \operatorname{End}\left(A^{n}\right)$ and $c b \in$ $\operatorname{rad} \operatorname{End}\left(B^{m}\right)$.

(ii) If $b c \in \operatorname{rad} \operatorname{End}\left(A^{n}\right)$ and $c b \in \operatorname{rad} \operatorname{End}\left(B^{m}\right)$, then there are idempotents $e_{1} \in \operatorname{End}\left(A^{n}\right)$ and $e_{2} \in \operatorname{End}\left(B^{m}\right)$ such that $a^{\prime}:=a-e_{1} \in \operatorname{rad} \operatorname{End}\left(A^{n}\right)$, $d^{\prime}:=d-e_{2} \in \operatorname{rad} \operatorname{End}\left(B^{m}\right)$ and $e(X) \cong e_{1}\left(A^{n}\right) \oplus e_{2}\left(B^{m}\right)$, where $e(X)$ denotes the direct summand of $X$ given by the idempotent $e$.

Proof. The first claim is obvious because $b c: A^{n} \rightarrow B^{m} \rightarrow A^{n}$ and $A$, $B$ are nonisomorphic indecomposable objects.

For the second, we notice first that $e^{2}=e$ implies $a^{2}+b c=a$ and $d^{2}+c b=d$, hence

$$
a^{2} \equiv a \quad\left(\bmod \operatorname{rad} \operatorname{End}\left(A^{n}\right)\right), \quad d^{2} \equiv d \quad\left(\bmod \operatorname{rad} \operatorname{End}\left(B^{m}\right)\right) .
$$

Thus by the assumption, we can lift $a$ and $d$ to the idempotents $e_{1}$ and $e_{2}$ in $\operatorname{End}\left(A^{n}\right)$ and $\operatorname{End}\left(B^{m}\right)$ respectively. Setting $\epsilon=\left(\begin{array}{cc}e_{1} & 0 \\ 0 & e_{2}\end{array}\right)$ and $\gamma:=$ $e-\epsilon=\left(\begin{array}{ll}a^{\prime} & b \\ c & d^{\prime}\end{array}\right)$, we see that

$$
\gamma^{2}=\left(\begin{array}{cc}
a^{\prime 2}+b c & a^{\prime} b+b d^{\prime} \\
c a^{\prime}+d^{\prime} c & c b+d^{\prime 2}
\end{array}\right) \in \operatorname{rad} \operatorname{End}(X)
$$

since $a^{\prime}, b c \in \operatorname{rad} \operatorname{End}\left(A^{n}\right)$ and $d^{\prime}, c b \in \operatorname{rad} \operatorname{End}\left(B^{m}\right)$. Hence $1-\gamma^{2}=$ $(1+\gamma)(1-\gamma)$ is invertible in $\operatorname{End}(X)$. Since $(1+\gamma) \epsilon=(\epsilon+\gamma) \epsilon=e \epsilon=$ $e(e-\gamma)=e(1-\gamma)$, we have $e=(1+\gamma) \epsilon(1-\gamma)^{-1}$. It then follows that $e(X) \cong \epsilon(X)=e_{1}\left(A^{n}\right) \oplus e_{2}\left(B^{m}\right)$, which completes the proof.

Now we can prove Theorem 3.4. 
Proof. Suppose we are given an idempotent $e \in \operatorname{End}\left(X \widehat{\otimes} X^{\prime}\right)$. Then we can write

$$
e=\left(\left(\begin{array}{ll}
\alpha & \beta \\
\gamma & \delta
\end{array}\right),\left(\begin{array}{ll}
\alpha^{\prime} & \beta^{\prime} \\
\gamma^{\prime} & \delta^{\prime}
\end{array}\right)\right),
$$

where $\alpha, \alpha^{\prime}, \beta$ etc. are $n m \times n m$ matrices over $S$. Considering the reduction to $S /(y)$, we have from $(2.10)$ that $e_{y} \in \operatorname{End}\left(\left(X \widehat{\otimes} X^{\prime}\right)_{y}\right)=\operatorname{End}\left(X^{m} \oplus \Omega X^{m}\right)$ is an idempotent and we can describe $e_{y}$ as

$$
\left(\begin{array}{cc}
\left(\alpha, \alpha^{\prime}\right)_{y} & \left(\beta, \beta^{\prime}\right)_{y} \\
\left(\gamma, \gamma^{\prime}\right)_{y} & \left(\delta, \delta^{\prime}\right)_{y}
\end{array}\right) .
$$

Since $X \not \Omega X$, it follows from Lemma 3.5 that there are idempotents $e_{1}=\left(\epsilon_{1}, \epsilon_{1}^{\prime}\right) \in \operatorname{End}\left(X^{m}\right)$ and $e_{2}=\left(\epsilon_{2}, \epsilon_{2}^{\prime}\right) \in \operatorname{End}\left(\Omega X^{m}\right)$ such that $e_{y}((X \widehat{\otimes}$ $\left.\left.X^{\prime}\right)_{y}\right) \cong e_{1}\left(X^{m}\right) \oplus e_{2}\left(\Omega X^{m}\right)$ that is isomorphic to $X^{k} \oplus \Omega X^{\ell}$ for some $0 \leqq k, \ell \leqq m$, because $X$ and $\Omega X$ are indecomposable. Note that by taking a suitable basis we may write $e_{1}=\left(\begin{array}{cc}1_{k} & 0 \\ 0 & 0\end{array}\right)$ as a morphism $X^{m} \rightarrow X^{m}$ and $e_{2}=\left(\begin{array}{cc}1_{\ell} & 0 \\ 0 & 0\end{array}\right)$ as a morphism $\Omega X^{m} \rightarrow \Omega X^{m}$, where $1_{k}$ (resp. $1_{\ell}$ ) denotes the identity morphism on $X^{k}$ (resp. $\Omega X^{\ell}$ ). Since Lemma (3.5) shows that $\left(\alpha, \alpha^{\prime}\right)_{y}-e_{1} \in \operatorname{rad} \operatorname{End}\left(X^{m}\right)$, further change of basis makes $\left(\alpha, \alpha^{\prime}\right)_{y}=\left(\begin{array}{cc}1_{k} & 0 \\ 0 & A\end{array}\right)$ for some $A \in \operatorname{rad} \operatorname{End}\left(X^{m-k}\right)$. Similarly we may get $\left(\delta, \delta^{\prime}\right)_{y}=\left(\begin{array}{cc}1_{\ell} & 0 \\ 0 & D\end{array}\right)$ for some $D \in \operatorname{rad} \operatorname{End}\left(\Omega X^{m-\ell}\right)$. Thus finally we can make $e_{y}$ into the following form as a morphism from $X^{k} \oplus X^{m-k} \oplus \Omega X^{\ell} \oplus$ $\Omega X^{m-\ell}$ into itself:

$$
e_{y}=\left(\begin{array}{cc}
\left(\alpha, \alpha^{\prime}\right)_{y} & \left(\beta, \beta^{\prime}\right)_{y} \\
\left(\gamma, \gamma^{\prime}\right)_{y} & \left(\delta, \delta^{\prime}\right)_{y}
\end{array}\right) \cong\left(\begin{array}{cccc}
1_{k} & 0 & 0 & 0 \\
0 & A & 0 & B \\
0 & 0 & 1_{\ell} & 0 \\
0 & C & 0 & D
\end{array}\right)
$$

for some $B$ and $C$. Therefore we have $e_{y}\left(\left(X \widehat{\otimes} X^{\prime}\right)_{y}\right) \cong X^{k} \oplus \Omega X^{\ell} \oplus$ Image $\left(\begin{array}{ll}A & B \\ C & D\end{array}\right)$, hence $\left(\begin{array}{ll}A & B \\ C & D\end{array}\right)=0$. This shows that $\left(\alpha, \alpha^{\prime}\right)_{y}$ itself is an epimorphism from $X^{m}$ to a direct summand $X^{k}$ of $X^{m}$, hence

$$
\operatorname{rank}\left(\alpha \otimes_{S} K\right)=\operatorname{rank}\left(\alpha^{\prime} \otimes_{S} K\right)=n k
$$


Likewise we see

$$
\operatorname{rank}\left(\delta \otimes_{S} K\right)=\operatorname{rank}\left(\delta^{\prime} \otimes_{S} K\right)=n \ell
$$

Now considering the reduction to $S / x S$, we have

$$
e_{x}=\left(\begin{array}{cc}
\left(\alpha, \delta^{\prime}\right)_{x} & \left(\beta,-\gamma^{\prime}\right)_{x} \\
\left(\gamma,-\beta^{\prime}\right)_{x} & \left(\delta, \alpha^{\prime}\right)_{x}
\end{array}\right)
$$

and $e_{x}\left(\left(X \otimes X^{\prime}\right)_{x}\right) \cong X^{\prime p} \oplus \Omega X^{\prime q}$ for some $0 \leqq p, q \leqq n$. Thus by the same argument as above we have

$$
\operatorname{rank}\left(\alpha \otimes_{S} K\right)=\operatorname{rank}\left(\delta^{\prime} \otimes_{S} K\right)=m p
$$

and

$$
\operatorname{rank}\left(\delta \otimes_{S} K\right)=\operatorname{rank}\left(\alpha^{\prime} \otimes_{S} K\right)=m q .
$$

Therefore from $(i)$ to $(i v)$ above we see that $n k=m p=n \ell=m q$, hence $k=\ell$ and $p=q$. As a consequence the size of the matrix factorization $e\left(X \widehat{\otimes} X^{\prime}\right)$ is $n k+n \ell=2 n k$ which also equals to $m p+m q=2 m p$, hence it is $\geqq \operatorname{lcm}(2 n, 2 m)=2 n m / r$. Since we have shown that every direct summand of $X \widehat{\otimes} X^{\prime}$ have size at least $2 n m / r$ and since the size of $X \widehat{\otimes} X^{\prime}$ is $2 n m$, we have $\#\left(X \widehat{\otimes} X^{\prime}\right) \leqq 2 n m /(2 n m / r)=r$ as desired.

Corollary 3.6. Suppose $r=1$. Then, (i) $X \widehat{\otimes} X^{\prime}$ is either indecomposable or decomposed into two indecomposable factors.

(i) If $\Omega X \not \nexists X$ and if $\Omega X^{\prime} \not X^{\prime}$, then $X \widehat{\otimes} X^{\prime}$ is indecomposable.

If one of $X$ and $X^{\prime}$ has size 1, then we have a stronger claim for the indecomposability of $X \widehat{\otimes} X^{\prime}$ than Theorem 3.4.

THEOREM 3.7. Adding to the assumption in (3.1), suppose $m=1$ and $\Omega\left(X^{\prime}\right) \not X^{\prime}$. Then the tensor product $X \widehat{\otimes} X^{\prime}$ is also indecomposable. In other words, the functor ( ) $\widehat{\otimes} X^{\prime}$ preserves the indecomposability.

Proof. Since $X^{\prime}$ has size one, we may write $X^{\prime}=(u, v)$ for some $u, v \in$ $K[[y]]$. Then the assumption $\Omega\left(X^{\prime}\right) \not X^{\prime}$ means exactly that $u K[[y]] \neq$ $v K[[y]]$. In the following we assume

$$
u \notin v K[[y]]
$$


(In case of $v \notin u K[[y]]$, an argument similar to below will prove the theorem.) First we remark that for any morphism $(a, b): X^{\prime n} \rightarrow \Omega\left(X^{\prime}\right)^{n}$ and $(c, d): \Omega\left(X^{\prime}\right)^{n} \rightarrow X^{\prime n}$, we have

$$
a \otimes_{K[[y]]} K=d \otimes_{K[[y]]} K=0 .
$$

To show this, supposed $a \otimes K \neq 0$. Then $a$ would be a matrix over $K[[y]]$ containing at least one unit element. Since $a u=v b$, we would have $u \in$ $v K[[y]]$ contradicting $(i)$.

Now let $Y=X \widehat{\otimes} X^{\prime}$, and suppose that $e=\left(\left(\begin{array}{ll}\alpha & \beta \\ \gamma & \delta\end{array}\right),\left(\begin{array}{ll}\alpha^{\prime} & \beta^{\prime} \\ \gamma^{\prime} & \delta^{\prime}\end{array}\right)\right)$ is an idempotent in $\operatorname{End}(Y)$. We want to show that $e=1$ or $e=0$. Note first that we may assume that $X \cong \Omega(X)$, since otherwise we have already shown in (3.6) that $Y$ is indecomposable.

As in the previous proof consider the reduction to $S / x S$, and we see from (2.10) that $e_{x}=\left(\begin{array}{cc}\left(\alpha, \delta^{\prime}\right)_{x} & \left(\beta,-\gamma^{\prime}\right)_{x} \\ \left(\gamma,-\beta^{\prime}\right)_{x} & \left(\delta, \alpha^{\prime}\right)_{x}\end{array}\right)$ is an idempotent on $Y_{x} \cong X^{\prime n} \oplus$ $\Omega\left(X^{\prime}\right)^{n}$, where $\left(\beta,-\gamma^{\prime}\right)_{x}: \Omega\left(X^{\prime}\right)^{n} \rightarrow X^{\prime n}$ and $\left(\gamma,-\beta^{\prime}\right)_{x}: X^{\prime n} \rightarrow \Omega\left(X^{\prime}\right)^{n}$. It then follows from $(i i)$ that $\gamma_{x} \otimes K=\gamma_{x}^{\prime} \otimes K=0$, that is,

$$
\text { all entries of } \gamma \text { and } \gamma^{\prime} \text { are non units in } S=K[[x, y]] \text {. }
$$

Considering the reduction to $y$ we see that $e_{y}=\left(\begin{array}{cc}\left(\alpha, \alpha^{\prime}\right)_{y} & \left(\beta, \beta^{\prime}\right)_{y} \\ \left(\gamma, \gamma^{\prime}\right)_{y} & \left(\delta, \delta^{\prime}\right)_{y}\end{array}\right)$ is an idempotent on $Y_{y}=X \oplus \Omega(X)$ since $m=1$. Setting $\Lambda=\operatorname{End}(X)=$ $\operatorname{End}(\Omega(X))$ that is a local ring, we see from $(i i i)$ that $\left(\beta, \beta^{\prime}\right)_{y}\left(\gamma, \gamma^{\prime}\right)_{y}=$ $\left(\beta \gamma, \beta^{\prime} \gamma^{\prime}\right)_{y} \in \operatorname{rad} \Lambda$, and $\left(\gamma, \gamma^{\prime}\right)_{y}\left(\beta, \beta^{\prime}\right)_{y} \in \operatorname{rad} \Lambda$. Then Lemma 3.4 implies that $\left(\alpha, \alpha^{\prime}\right)_{y}$ and $\left(\delta, \delta^{\prime}\right)_{y}$ will give idempotents in $\Lambda / \operatorname{rad} \Lambda$. Since $\Lambda$ is local, we see that $\left(\alpha, \alpha^{\prime}\right)_{y}$ is either an automorphism on $X$ or an element in $\operatorname{rad} \Lambda$, and the same is true for $\left(\delta, \delta^{\prime}\right)_{y}$.

For the first case to be considered, assume $\left(\alpha, \alpha^{\prime}\right)_{y}$ is an automorphism on $X$. In this case, $\alpha$ and $\alpha^{\prime}$ are invertible matrices on $S$. Since $\left(\alpha, \delta^{\prime}\right)_{x}$ is a morphism $X^{\prime n} \rightarrow X^{\prime n}$, we have $\alpha_{x} u=u \delta_{x}^{\prime}$ hence $\delta_{x}^{\prime}$ is also an invertible matrix on $K[[y]]$. Therefore $\delta^{\prime}$ is also invertible as a matrix on $S$. Similarly considering $\left(\delta, \alpha^{\prime}\right)_{x}$, we see that $\delta$ is an invertible matrix on $S$. Combining these result with $(i i i)$ we show that the matrices $\left(\begin{array}{ll}\alpha & \beta \\ \gamma & \delta\end{array}\right)$ and $\left(\begin{array}{ll}\alpha^{\prime} & \beta^{\prime} \\ \gamma^{\prime} & \delta^{\prime}\end{array}\right)$ are invertible. Then it follows that the idempotent $e$ is an automorphism, hence $e=1$.

In the case that $\left(\delta, \delta^{\prime}\right)_{y}$ is an automorphism, the similar argument as above leads $e=1$. 
Finally consider the case that both of $\left(\alpha, \alpha^{\prime}\right)_{y}$ and $\left(\delta, \delta^{\prime}\right)_{y}$ are in $\operatorname{rad} \Lambda$. In this case we have from (iii) that $e_{y} \otimes_{\Lambda} \Lambda / \operatorname{rad} \Lambda=\left(\begin{array}{cc}0 & \left(\beta, \beta^{\prime}\right)_{y} \\ 0 & 0\end{array}\right)$ is an idempotent, hence $\left(\beta, \beta^{\prime}\right)_{y} \in \operatorname{rad} \Lambda$. As a consequence we have $e_{y}=0$, from which we see that all the entries of $\alpha, \beta, \alpha^{\prime}$ etc. are in $y S$. Since $e$ is an idempotent, it then follows that $e=0$. This completes the proof.

EXAMPLE 3.8. Let $K$ be an algebraically closed field of characteristic $\neq 2$ and let $n_{i}=r_{i}+s_{i}(i=1,2, \ldots, \ell)$ be natural numbers. Then $Y=$ $\left(\left(x_{1}^{r_{1}}, x_{1}^{s_{1}}\right) \widehat{\otimes} \cdots \widehat{\otimes}\left(x_{\ell}^{r_{\ell}}, x_{\ell}^{s_{\ell}}\right)\right)$ is a matrix factorization in $M F\left(x_{1}^{n_{1}}+\ldots+x_{\ell}^{n_{\ell}}\right)$. Putting the integer part of $\frac{1}{2} \#\left\{i \mid r_{i}=s_{i}\right\}$ as $u$, we have $\#(Y)=2^{u}$.

\section{$\S 4$. An application}

In this section let $R=K[[x]] /(f(x))=K\left[\left[x_{1}, x_{2}, \ldots, x_{s}\right]\right] /\left(f\left(x_{1}, x_{2}, \ldots\right.\right.$, $\left.x_{s}\right)$ ) be a hypersurface ring. For a given sequence of integers $\left\{n_{1}, n_{2}, \ldots, n_{r}\right\}$ we may consider the ring $R_{r}=K\left[\left[x, y_{1}, y_{2}, \ldots, y_{r}\right]\right] /\left(f(x)+y_{1}^{n_{1}}+y_{2}^{n_{2}}+\ldots+\right.$ $y_{r}^{n_{r}}$ ). Of course there is a natural ring homomorphism $R_{r} \rightarrow R$ (sending $y_{i}$ to 0 ), through which every $R$-module can be regarded as an $R_{r}$-module. Particularly, for any MCM module over $R$, we can take the $r$-th syzygy $\Omega_{R_{r}}^{r}(M)$ of $M$ as an $R_{r}$-module, which is an MCM module over $R_{r}$. If $M$ is indecomposable and if $n_{1}=n_{2}=\ldots=n_{r}=2$, then Knörrer [K2] shows how $\Omega_{R_{r}}^{r}(M)$ decomposes. To the contrary, if all of $n_{i}$ are greater than 2, then we can show that the functor $\Omega_{R_{r}}^{r}$ preserves the indecomposability.

THEOREM 4.1. Under the above notation, assume that $n_{i} \geqq 3$ for $1 \leqq$ $i \leqq r$. If $M$ is an indecomposable MCM module over $R$, then the syzygy module $\Omega_{R_{r}}^{r}(M)$ is also indecomposable.

We denote the minimal CM approximation of $M$ as an $R_{r}$-module by $X^{R_{r}}(M)$ which was defined by Auslander and Buchweitz [AB]. Notice that, in our case, $X^{R_{r}}(M) \cong \Omega_{R_{r}}^{r}\left(M^{*}\right)^{\prime}$ where ( )* (resp. ( $\left.)^{\prime}\right)$ denotes the $R$-dual (resp. $R_{r}$-dual). Since the duality preserves the indecomposability of MCM modules, the above theorem is equivalent to the following one.

THEOREM 4.2. Assume $n_{i} \geqq 3$ for $1 \leqq i \leqq r$. Then $X^{R_{r}}(M)$ is indecomposable, whenever $M$ is an indecomposable MCM module over $R$.

Proof. First we consider the case $r=1$.

Since $M$ is an MCM module, as we remarked in the beginning of $\S 1$, there is a matrix factorization $(\phi, \psi) \in M F(f)$ such that $M$ has a minimal 
$R$-free resolution of the form:

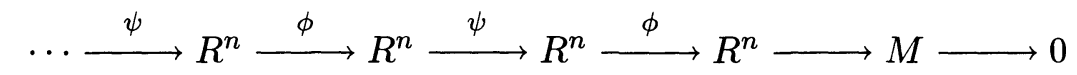

Note that $R=R_{1} /\left(y_{1}\right)$ and that $y_{1}$ is a non-zero divisor on $R_{1}$. And we can consider a lifting problem of complexes over $R$ onto $R_{1}$. In this case, since $\phi \cdot \psi=\psi \cdot \phi=f=-y_{1}\left(y_{1}^{n_{1}-1}\right)$ in $R_{1}$, we see that the Eisenbud operator of the complex $(i)$ with respect to $R_{1} \rightarrow R$ is given by $-y_{1}^{n_{1}-1}$. See [Y2] for the definition and the properties of Eisenbud operators. Then the L-complex defined in [Y2] is

$$
\cdots \longrightarrow R_{1}^{2 n} \stackrel{\left(\begin{array}{cc}
\psi & y_{1}^{n_{1}-1} \\
y_{1} & -\phi
\end{array}\right)}{\longrightarrow} R_{1}^{2 n} \stackrel{\left(\begin{array}{cc}
\phi & y_{1}^{n_{1}-1} \\
y_{1} & -\psi
\end{array}\right)}{\longrightarrow} R_{1}^{2 n} \stackrel{\left(y_{1}-\phi\right)}{\longrightarrow} R_{1}^{n} \longrightarrow M \longrightarrow 0,
$$

which actually resolves the module $M$ over $R_{1}$ as proved in [Y2, Lemma (2.3)].

Putting $Z:=(\psi, \phi) \in M F(f)$ and $Z^{\prime}:=\left(y_{1}, y_{1}^{n_{1}-1}\right) \in M F\left(y_{1}^{n_{1}}\right)$, we deduce from (ii) that $\Omega_{R_{1}}^{1}(M)$ is given by the matrix factorization $Z \widehat{\otimes} Z^{\prime}$.

Notice that $\Omega\left(Z^{\prime}\right) \not Z^{\prime}$ since $n_{1}-1>1$ and we can conclude from Theorem 3.7 that $Z \widehat{\otimes} Z^{\prime}$, hence $\Omega_{R_{1}}^{1}\left(X^{\prime}\right)$, is indecomposable.

Thus the proof of Theorem (4.1), hence (4.2), is completed for the case $r=1$.

The rest of the proof will be done by the induction on $r$. Now let $r>1$ and set $X:=X^{R_{r}}\left(X^{R_{r-1}}(M)\right)$. By the induction hypothesis, $X$ is indecomposable. Therefore if we show $X \cong X^{R_{r}}(M)$, then it completes the proof. By the definition of CM approximation we have the exact sequences:

$$
\begin{aligned}
& 0 \longrightarrow Y^{R_{r-1}}(M) \longrightarrow X^{R_{r-1}}(M) \stackrel{p}{\longrightarrow} M \longrightarrow 0, \\
& 0 \longrightarrow Y:=Y^{R_{r}}\left(X^{R_{r-1}}(M)\right) \longrightarrow X \stackrel{q}{\longrightarrow} X^{R_{r-1}}(M) \longrightarrow 0,
\end{aligned}
$$

where $Y$ (resp. $\left.Y^{R_{r-1}}(M)\right)$ is of finite projective dimension over $R_{r}$ (resp. over $R_{r-1}$.) Notice that $Y^{R_{r-1}}(M)$ has also finite projective dimension regarded as a module over $R_{r}$, since $R_{r-1}=R_{r} /\left(y_{r}\right)$ and $y_{r}$ is a non-zero divisor on $R_{r}$. Now let $Z$ be the kernel of the composite $p \cdot q: X \rightarrow M$ and one can show that there is an exact sequence $0 \rightarrow Y \rightarrow$ $Z \rightarrow Y^{R_{r-1}}(M) \rightarrow 0$. Therefore $Z$ is a module of finite projective dimension over $R_{r}$. Hence the sequence $0 \rightarrow Z \rightarrow X \stackrel{p q}{\rightarrow} M \rightarrow 0$ is a CM approximation 
of $M$. It then follows from the definition that $X^{R_{r}}(M)$ is a direct summand of $X$. Since we know that $X$ is indecomposable, we have $X^{R_{r}}(M)=X$ as desired.

Remark 4.3. The same argument as in the above proof can be applied in several other cases. For example one can prove the following claim:

Let $R=K\left[\left[x_{1}, \ldots, x_{r}, y_{0}, y_{1}, \ldots, y_{s}\right]\right] /\left(f(x)+y_{0} g(y)\right)$ and let $\bar{R}=$ $R /\left(y_{0}\right)$. Suppose that an MCM module $M$ over $\bar{R}$ is indecomposable. Then, in general, $\Omega_{R}^{1}(M)$ decomposes into at most two factors. If $y_{0} K[[y]] \neq$ $g(y) K[[y]]$, then $\Omega_{R}^{1}(M)$ is indecomposable.

It is also easy to see that the same is true for $X^{R}(M)$ instead of $\Omega_{R}^{1}(M)$.

\section{REFERENCES}

[AB] M. Auslander and R. O. Buchweitz, The homological theory of maximal Cohen-Macaulay approximations, Soc. Math. de France, Mem., 38 (1989), 5-37.

[ADS] M. Auslander, S. Ding and $\varnothing$. Solberg, Liftings and weak liftings of modules, J. Algebra, 156 (1993), 273-317.

[BEH] R.-O. Buchweitz, D. Eisenbud and J. Herzog, Cohen-Macaulay modules on quadrics, Singularities, representation of algebras and vector bundles (Lambrecht, 1985), Springer Lecture Notes in Math., 1273 (1987), pp. 58-116.

[E] D. Eisenbud, Homological algebra on a complete intersection, with an application to group representations, Trans. A. M. S., 260 (1980), 35-64.

[HP] J. Herzog and D. Popescu, Thom-Sebastiani problems for maximal CohenMacaulay modules, to appear in Math. Ann..

[K1] H. Knörrer, The Cohen-Macaulay modules over simple hypersurface singularities, Representation of algebras, London Math. Soc., 116 (1985), 147-164.

[K2] H. Knörrer, Cohen-Macaulay modules on hypersurface singularities I, Invent. Math., 88 (1987), 153-164.

[S] Ø. Solberg, Hypersurface singularities of finite Cohen-Macaulay type, Proc. London Math. Soc., 58 (1989), 258-280.

[Y1] Y. Yoshino, Cohen-Macaulay modules over Cohen-Macaulay rings, Lecture Note Series of London Math. Soc., vol. 146, Cambridge University Press, Cambridge, 1990.

[Y2] Y. Yoshino, The theory of L-complexes and weak liftings of complexes, J. Algebra, 188 (1997), 144-183.

Institute of Math.,

Faculty of Integrated Human Studies, Kyoto Univ.

Yoshida-Nihonmatsu, Sakyo-ku,

Kyoto 606-01, Japan

yoshino@math.h.kyoto-u.ac.jp 